\title{
Reynolds-Averaged Navier-Stokes Simulation of a 2D Circulation Control Wind Tunnel Experiment
}

\author{
Brian G. Allan, Greg S. Jones† John C. Lin ${ }^{\ddagger}$ \\ NASA Langley Research Center, Hampton, VA
}

\begin{abstract}
Numerical simulations are performed using a Reynolds-averaged Navier-Stokes (RANS) flow solver for a circulation control airfoil. 2D and 3D simulation results are compared to a circulation control wind tunnel test conducted at the NASA Langley Basic Aerodynamics Research Tunnel (BART). The RANS simulations are compared to a low blowing case with a jet momentum coefficient, $C_{\mu}$, of 0.047 and a higher blowing case of 0.115 . Three dimensional simulations of the model and tunnel walls show wall effects on the lift and airfoil surface pressures. These wall effects include a $4 \%$ decrease of the midspan sectional lift for the $C_{\mu} 0.115$ blowing condition. Simulations comparing the performance of the Spalart Allmaras (SA) and Shear Stress Transport (SST) turbulence models are also made, showing the SST model compares best to the experimental data. A Rotational/Curvature Correction (RCC) to the turbulence model is also evaluated demonstrating an improvement in the CFD predictions.
\end{abstract}

\section{Nomenclature}

\begin{tabular}{llll} 
BART & Basic Aerodynamics Research Tunnel & $\dot{m}$ & Mass flow rate \\
CC & Circulation Control & $N P R$ & Nozzle Pressure Ratio, $p_{t} / p_{\infty}$ \\
CFD & Computational Fluid Dynamics & $p$ & pressure \\
GTRI & Georgia Tech Research Institute & $p_{t}$ & total pressure \\
LMP & Low-Mach number Preconditioning & $q$ & dynamic pressure, $\frac{1}{2} \rho U^{2}$ \\
NASA & National Aeronautics and Space & $S$ & Reference area, $b c$ \\
& Administration & $u, v, w$ & Velocity in $x, y, z$ directions \\
RANS & Reynolds-averaged Navier-Stokes & & respectively \\
RCC & Rotational/Curvature Correction & $\vec{V}$ & Velocity vector, $(\mathrm{u}, \mathrm{v}, \mathrm{w})$ \\
& to turbulence model & $x$ & Streamwise axis \\
RE & Richardson Extrapolation & $y$ & Vertical axis \\
$\alpha$ & angle of attack, deg. & $z$ & Spanwise axis \\
$b$ & span $(28.0 i n)$. & & \\
$c$ & chord length $(8.6 \mathrm{in})$. & \multicolumn{2}{c}{ Subscripts } \\
$C_{L}$ & lift force coef., $L /\left(q_{\infty} c b\right)$ & $\infty$ & Freestream \\
$C_{\mu}$ & jet momentum coeff. $=\dot{m} u_{j} /\left(q_{\infty} S\right)$ & $j$ & Jet \\
$C_{p}$ & pressure coef., $\left(p-p_{\infty}\right) /\left(q_{\infty} c b\right)$ & &
\end{tabular}

*Research Scientist, Flow Physics and Controls Branch, NASA Langley Research Center, Mail Stop 170, Hampton VA 23681-2199. AIAA Senior Member

†Senior Scientist, Flow Physics and Controls Branch, NASA Langley Research Center, Mail Stop 170, Hampton VA $23681-$ 2199. AIAA Senior Member

${ }^{\ddagger}$ Senior Scientist, Flow Physics and Controls Branch, NASA Langley Research Center, Mail Stop 170, Hampton VA $23681-$ 2199. AIAA Associate Fellow 


\section{Introduction}

$\mathrm{T}_{\mathrm{m}}^{\mathrm{H}} \mathrm{de}$ HE design of effective circulation control (CC) configurations require accurate and efficient prediction methods. To assess the capability of computational fluid dynamics (CFD) to predict CC airfoil flows, a CC workshop was held at NASA Langley in 2004. ${ }^{1}$ One of the outcomes from this workshop was that the lift performance of a benchmark CC airfoil case was greatly over predicted by almost all of the CFD codes. It was also found that the turbulence models played a critical role in the over prediction of lift where the jet detachment location on the Coanda surface was typically delayed as compared to experiments. Research has shown that by forcing the flow to separate on the Coanda surface, at the same location as observed in the experiment, that the CFD will typically match the experimental lift. Therefore, prediction of the correct separation location on the Coanda surface is synonymous with a CFD codes ability to predict the correct $\mathrm{CC}$ airfoil lift. Current research is showing that the RANS turbulence models maybe under predicting the eddy viscosity resulting in a $\mathrm{CC}$ jet that remains attached longer to the Coanda surface, thus over predicting the lift. ${ }^{2}$

In an effort to improve CFD codes for CC airfoils, NASA has initiated wind tunnel experiments to generate benchmark quality experimental data. This effort focuses on a simple CC airfoil geometry that was tested in both the separation control and super-circulation control regimes. Measurements for this experiment also focused on characterizing critical boundary conditions required for CFD code validation. ${ }^{3-5}$

Historically code validation comparing 2D CFD to "2D" wind tunnel test data has been problematic because the physics are inherently 3D and typically are influenced by wall effects. While there are simple angle of attack and freestream dynamic pressure corrections to approximate wall effects, they are typically used for low to moderate lift configurations and are inaccurate for airfoils with very large lift coefficients. To remove the ambiguity of comparing 2D CFD to CC wind tunnel data with wall corrections, this study will model the tunnel and model geometry in 3D, thereby simulating the actual wind tunnel experiment. The results from these $3 \mathrm{D}$ simulations will be used to help quantify wall effects and to compare against recent benchmark CC experimental data., ${ }^{4,5}$

\section{Numerical Modeling Approach}

\section{A. Flow Solver}

The flow field for the circulation controlled airfoil was computed using the flow solver code, OVERFLOW ${ }^{6,7}$ developed at NASA. This code solves the compressible RANS equations using the diagonal scheme of Pulliam and Chaussee. ${ }^{8}$ The RANS equations are solved on structured grids using the overset grid framework of Steger, Dougherty, and Benek. ${ }^{9}$ This overset grid framework allows for the use of structured grids for problems that have complex geometries and moving bodies. To improve the convergence and accuracy of the flow solution, the OVERFLOW code includes a Low-Mach number Preconditioning (LMP) option. The numerical simulations were performed using the parallel version of the OVERFLOW code developed by Buning. ${ }^{10}$ This code uses the Message-Passing Interface and can run on a tightly-coupled parallel machine or a network of workstations. The code distributes zones to individual processors and can split larger individual zones across multiple processors using a domain decomposition approach.

The RANS equations are solved implicitly using the Beam-Warming block tridiagonal scheme with a $3^{\text {nd }}$ order Roe upwind scheme for the inviscid flux terms. The Shear Stress Transport (SST) ${ }^{11}$ and Spalart Allmaras $(\mathrm{SA})^{12}$ turbulence models were both used assuming fully turbulent flow including inside the plenums and nozzle. A Rotational/Curvature Correction (RCC) model, as implemented in OVERFLOW, was evaluated for both turbulence models. ${ }^{13}$

\section{B. Grids}

The grids used for the 2D simulations are shown in Fig. 1 and are also used for the cross sections along the model span to build up the 3D grids. Figure 2 also shows the tunnel walls and contraction used in the 3D simulations. The structured overset grid system for both 2D and 3D were generated using the Chimera Grid Tools package. ${ }^{14}$

The airfoil grids have a total of 1241 grid points around the entire surface with 401 of these points concentrated on the Coanda trailing edge. There are 101 grid points in the viscous direction away from the airfoil surface within a distance of $0.23 c$, where $c$ is the airfoil chord. The Coanda trailing edge grid has 261 grid points in the viscous direction, extending $0.23 c$ from the surface. The large number of grid points on 


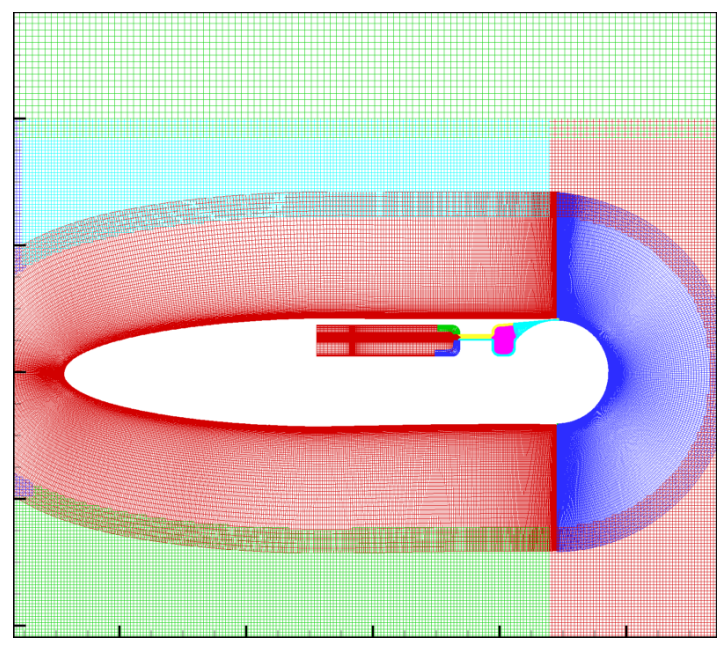

Figure 1. Grids used in the 2D simulations and for the cross sections along the model span for the 3D grids. This figure also shows the grids used to model the internal plenums.

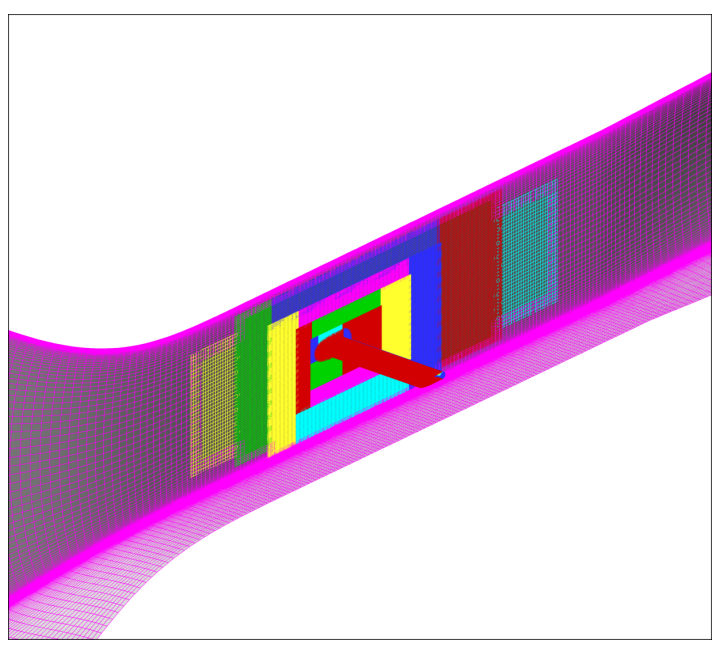

Figure 2. Surface grids for the 3D simulation that includes the tunnel walls and contraction. The 3D simulation also used a symmetry plane about the centerline of the tunnel.

the Coanda surface were used to resolve the evolution of the wall jet and the shear flow region on the outer edge of the jet.

The airfoil body grids are overset onto a single Cartesian background grid that has a uniform grid spacing of $0.0058 \mathrm{c}$ in all directions and extends $0.47 c$ upstream of the leading edge and downstream of the trailing edge. This is the finest Cartesian background grid and is referred to as a level 1 grid. The level 1 background grid is then overset onto a group of level 2 background grids that have double the cell size in all directions as the level 1 grid. The level of the Cartesian background grids are then doubling in cell size at each level until the far field boundaries are approximately 85 chord lengths away from the wing. The 2D simulations have approximately 500,000 grid points where 300,000 of these points were used for the body grids.

The 3D simulations have a symmetry plane at the centerline of the model with viscous grid spacing in the span direction at the wall juncture resulting in a total of 142 million grid points. The body grids and level 1 background grid, for the 3D case, have 309 points along the model span with 41 points clustered near the tunnel wall to resolve the tunnel wall boundary layer and juncture flows.

\section{Wind Tunnel Experiment}

The circulation control experiment for CFD validation was a collaborative effort between Georgia Tech Research Institute (GTRI) and NASA Langley. The goal of this collaborative research was to generate 2D benchmark quality data of a fundamental circulation control airfoil geometry for CFD validation. ${ }^{4}$ The CC model was developed and tested at GTRI with followup wind tunnel tests at NASA Langley. GTRI performed extensive testing of the overall performance and parameter characterization, while NASA is pursuing additional flow field measurements needed for CFD validation at the NASA Langley BART.

\section{A. BART Wind Tunnel}

The BART is an open-circuit, atmospheric wind tunnel and was designed for code validation efforts. ${ }^{15}$ The test section is $28 \mathrm{in}$. high by $40 \mathrm{in}$. wide and $10 \mathrm{ft}$ long with a maximum speed of approximately $220 \mathrm{ft} / \mathrm{s}$. The BART tunnel is also equipped with wall blowing (on the floor and ceiling), at the test section entrance, to decrease the effects of the juncture vortex by energizing the wall boundary layer. ${ }^{4}$ Wall pressures were also measured on the centerline of the sides walls, opposite the upper and lower surface of the CC model.

\section{B. CC Model}

A 2D section of the CC model is shown in Fig. 3 and has an elliptic leading edge with a large circular trailing edge. This model was chosen for benchmark testing because of its simple geometry and large trailing edge 


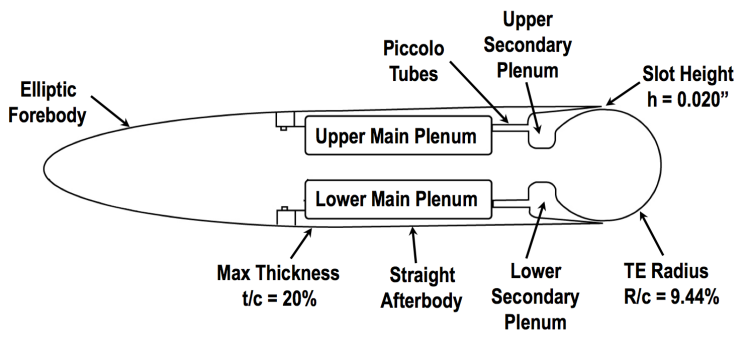

Figure 3. A 2D section of the CC model showing the geometry of the CC-E0020EJ airfoil and the upper and lower blowing slots and plenums.

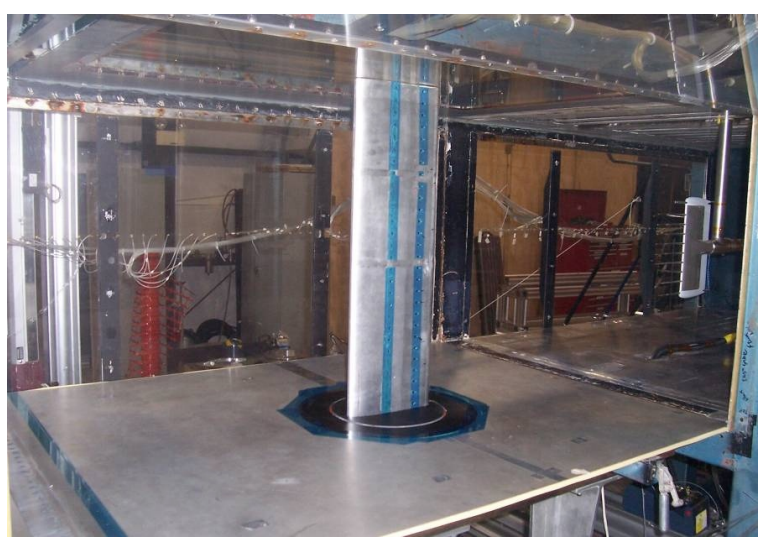

Figure 4. The 28 in. span $\mathrm{CC}$ model installation in the NASA BART tunnel mounted vertically with pressure taps on the centerline of the side walls.

radius. The large circular trailing edge is conducive to surface and near-body flow field measurements with improved accuracy as compared to CC airfoils with smaller trailing edge radii. The CC model has dual blowing slots on the upper and lower portions of the trailing edge; however, in this investigation, the lower slot was closed with only the upper slot open.

The CC model has a chord length of 8.6 in. and was mounted vertically in BART from floor to ceiling, as shown in Fig. 4, and has a span of $28 \mathrm{in.} \mathrm{The} \mathrm{model} \mathrm{has} \mathrm{an} \mathrm{aspect} \mathrm{ratio} \mathrm{(span/chord)} \mathrm{of} 3.26$ and a tunnel height to model chord ratio of 4.6. For the BART tests, the blowing slot was set to a constant height of 0.020 in $(0.00232 c)$. This slot configuration is not optimal for the maximum lift performance but was chosen for measurement resolution of the jet at the slot exit. The total pressure of the jet was measured using three total pressure probes along the span of the model in the secondary plenum. The total temperature and mass flow rate were measured in the air supply line external to the model.

\section{Results}

\section{A. Slot Jet Profile}

In the CFD simulations, the jet conditions are defined by using an inflow boundary condition at the first plenum chamber that sets the total pressure, $p_{t, \text { plenum }}$, and total temperature, $T_{t, \text { plenum }}$. The CFD simulation then solves the internal flow from the first plenum through a contraction to the second plenum into the nozzle and then slot exit. Figure 5 shows streamlines from a 2D simulation revealing the complex flow inside the second plenum. At this point it is unknown how much of the internal geometry needs to be modeled.

To verify the slot velocities from the CFD simulations, detailed hot-wire measurements were made at the slot exit with the model on a bench top (i.e., no external flow) and compared to 2D CFD simulation results. A close-up view of the slot exit is shown from a 2D CFD simulation in Fig. 6 with streamlines revealing a separation zone behind the slot lip.

Numerical simulations matching the two blowing conditions of the hot-wire measurements were made and compared to the experimental results in Fig. 7 and 8. Initially the CFD simulations included an external flow with a freestream Mach number of 0.1. The profiles from the CFD showed a significantly larger jet velocity for the same jet NPR and total temperature. Since the bench top measurements did not have an external flow, a second CFD simulation using a quiescent flow $(\mathrm{Mach}=0)$ condition was performed and resulted in an almost exact match of the measured slot velocity profile. Figure 7 shows a low-blowing case at an NPR $=1.10$ and Fig. 8 a higher-blowing case at NPR=1.21. The comparisons also show a good match of the slot height between the CFD and the experiment.

Currently the standard definition of the NPR uses the tunnel freestream static pressure, which is measured upstream at the tunnel contraction location. These results indicate that this freestream static pressure is not characteristic of the effective NPR for the jet velocity and that it should not be used to characterize the peak velocity.

Figures 9 and 10 show the static pressure ratio, $p / p_{\infty}$, and total pressure ratio, $p_{t} / p_{t, \text { plenum }}$, at the slot exit for the CFD simulation with $\mathrm{NPR}=1.21$. From these figures we see that the total pressure at the core of 


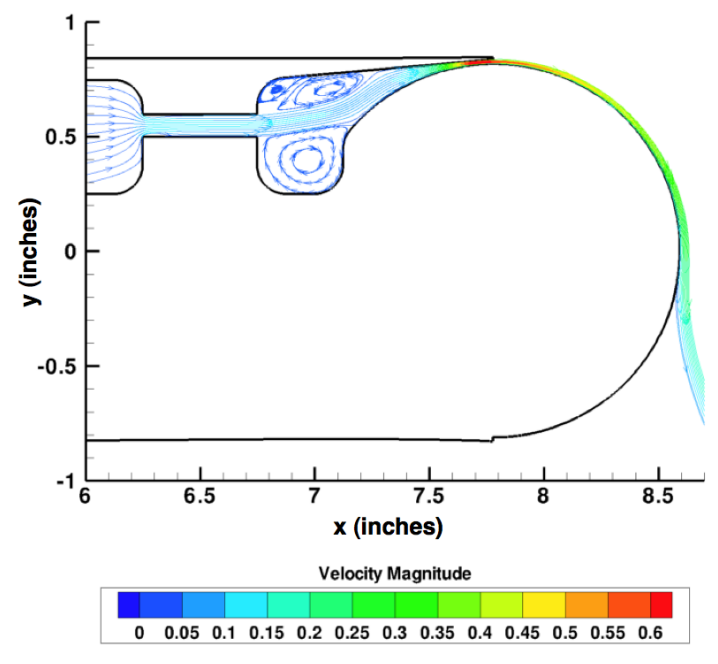

Figure 5. Streamlines shaded by the velocity magnitude nondimensionalized by freestream speed of sound. These streamlines highlight the complex flow inside the second internal plenum. The results are from a 2D CFD simulation using an NPR of 1.21 with a freestream Mach number of 0.1 .

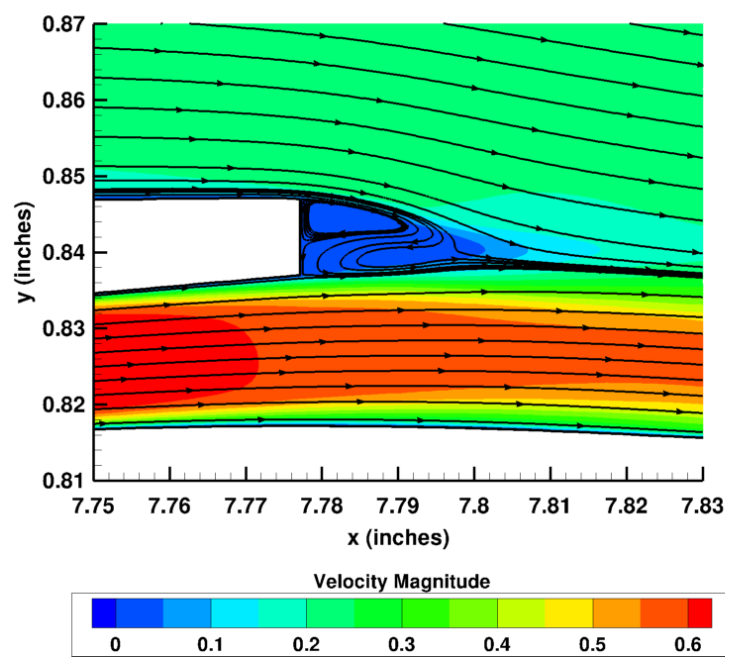

Figure 6. Contour plot of the velocity magnitude, nondimensionalized by freestream speed of sound, with streamlines highlighting the flow at the slot exit and the wake behind the thickness of the upper slot geometry. This plot is from a 2D CFD simulation of the circulation control airfoil with an NPR of 1.21 and a freestream Mach number of 0.1.

the slot is nearly equal to the plenum total pressure with and without external flow. The static pressure at the slot exit, with no external flow, is between 0.980 and 0.995 of the freestream value. Figure 9 shows that with external flow, the static pressure ratio is greatly reduced at the slot resulting in a higher jet velocity. Figures 11 and 12 are contour plots of the static pressure ratio near the slot with and without external flow. The airfoil with external flow generates lift accelerating the flow around the Coanda surface, lowering the static pressure near the slot exit. Therefore, the increased jet velocity seen in the CFD simulations is a result of the external flow reducing the static pressure near the slot, increasing the jet velocity.

While no slot velocity measurements of the CC model in the wind tunnel were made, it is hypothesized, based on the CFD results, that the actual peak jet velocity will be larger than the bench top measurements. Based on the results with and without external flow, it is recognized that the jet velocity profiles will also need to be measured in the wind tunnel with external flow, in order to validate the CFD at the jet exit. Once validated with external flow, the jet profile can be validated on the bench top and only a peak jet velocity at the exit will be required in the wind tunnel, assuming the velocity profiles are similar with and without external flow.

Another check on the jet velocity difference with and without external flow is to compare the slot mass flow rates from the bench top and wind tunnel experiments for varying NPR values. Figure 13 shows a comparison of the mass flow rates between the CFD and experiment, with and without external flow as a function of NPR. This figure confirms that both the experiment and CFD have higher mass flow rates at a given NPR with an external flow when compared to the bench top cases. The plot also shows that the CFD is matching the mass flow rates of the experiment very well for both the quiescent and external flow cases and supports the influence of the external flow on the peak slot velocity.

\section{B. 2D CFD Simulations}

Simulations in 2D were performed in "free-air" (i.e., no tunnel walls) with the far field boundaries 85 chord lengths away from the body. The simulations matched a freestream Mach number of 0.1 , the NPR for the internal plenum, and jet total temperature ratio.

The CFD simulations focused on two blowing conditions from the wind tunnel test, a low blowing case at $C_{\mu}=0.047$ and a higher blowing case at $C_{\mu}=0.115$. The low blowing case of $C_{\mu}=0.047$ is considered to be in the separation control regime while the higher blowing case of $C_{\mu}=0.115$ is at the onset of the supercirculation control regime. The airfoil is at $0^{\circ}$ for the $2 \mathrm{D}$ simulations and no attempt was made to correct for the downwash due to the tunnel wall effects. Below we will present the results for the $2 \mathrm{D}$ simulations 


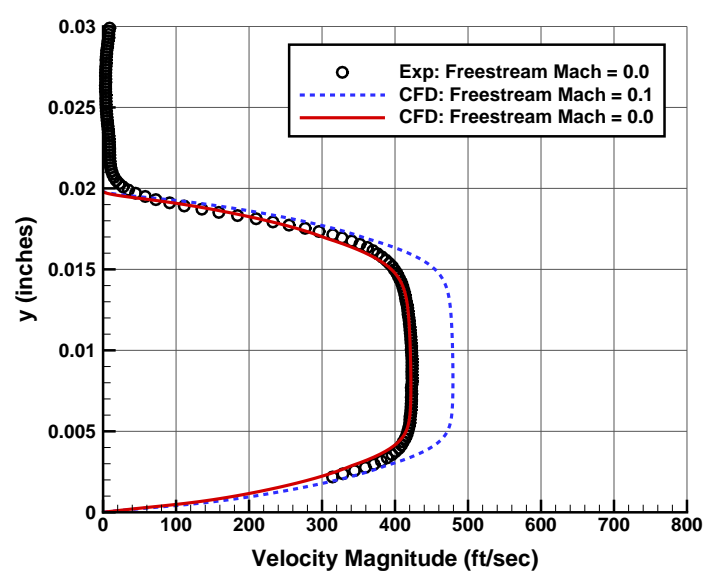

Figure 7. Exit velocity profiles for the circulation control model for NPR=1.10. Experimental data was measured on the bench top in quiescent flow and compared to CFD simulations with and without external flow.

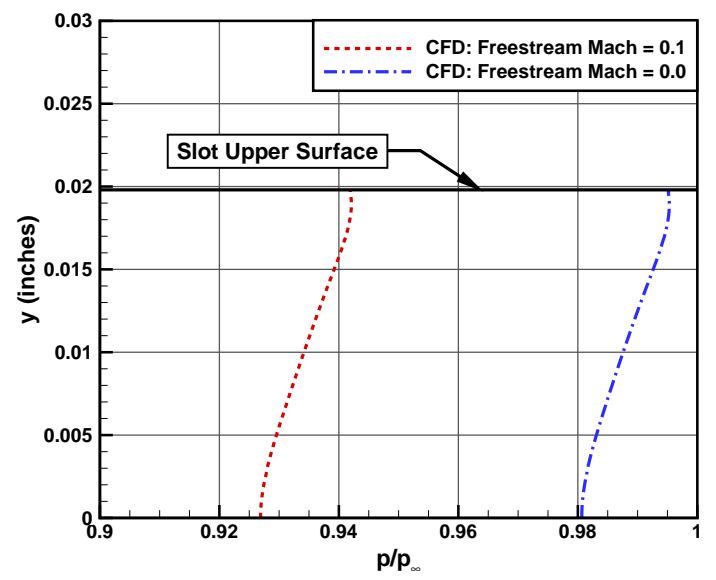

Figure 9. Jet static pressure ratio, $p / p_{\infty}$, profiles at the slot exit for the circulation control model for $\mathrm{NPR}=1.21$ with and without external flow.

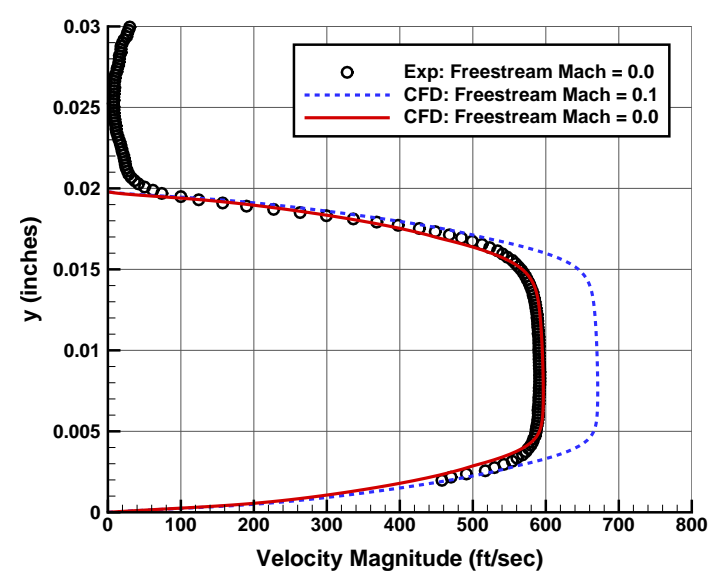

Figure 8. Exit velocity profiles for the circulation control model for $\mathrm{NPR}=1.21$. Experimental data was measured on the bench top in quiescent flow and compared to CFD simulations with and without external flow.

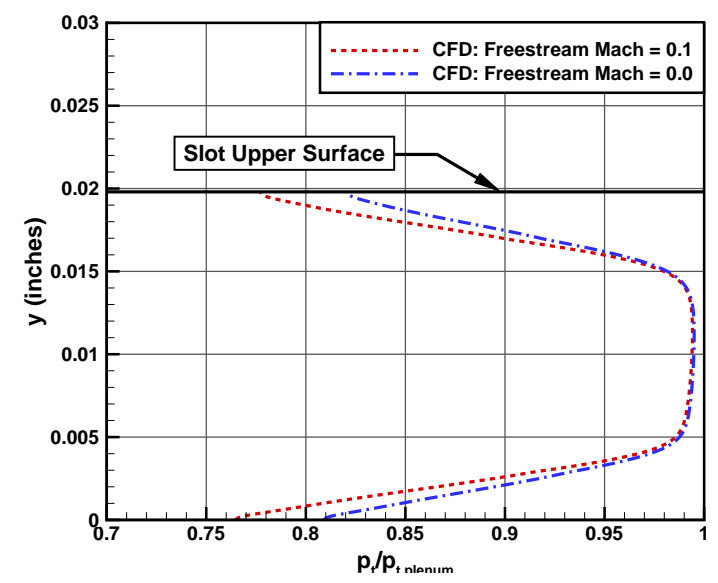

Figure 10. Jet total pressure ratio, $p_{t} / p_{t, p l e n u m}$, profiles at the slot exit for the circulation control model for $\mathrm{NPR}=1.21$ with and without external flow.

using the SA and SST turbulence models with and without LMP and RCC.

\section{1. $C_{\mu}=0.047$ Case}

A summary of the $C_{L}$ values for the low blowing case of $C_{\mu}=0.047$ is presented in Table 1 . The corresponding $C_{p}$ profiles are shown in Fig. 14 for the SA and SST turbulence models with and without RCC and LMP. The SA model without RCC predicted a lift coefficient at $C_{L}=2.03$ as compared to the experimentally measured value of 1.31 from the model balance. The experiment also had a sectional $C_{L}$ of 1.35 near the $3 / 4$ span at $y / b=0.73$ that was integrated from the pressure profile. Note that the sectional pressures near the midspan could not be used since this data was missing a critical pressure port on the upper surface of the model at $x / c=0.84$, resulting in an over prediction of $C_{L}$. Therefore, only the sectional $C_{L}$ near the $3 / 4$ span location could be used. Adding RCC to the SA model improved the lift results with a $C_{L}=1.86$; however, this is still $38 \%$ larger than the experimentally measured value. The SST model performed better with $C_{L}=1.58$ and was slightly improved by adding RCC where $C_{L}=1.56$. Turning on the LMP had little effect on the $C_{L}$ values and results in a small amount of unsteadiness of the lift convergence history.

While the suction pressure peak at the trailing edge is close to the experiment, the leading edge suction pressure peak is over predicted. It was noted that the $2 \mathrm{D}$ simulations are without an angle of attack 


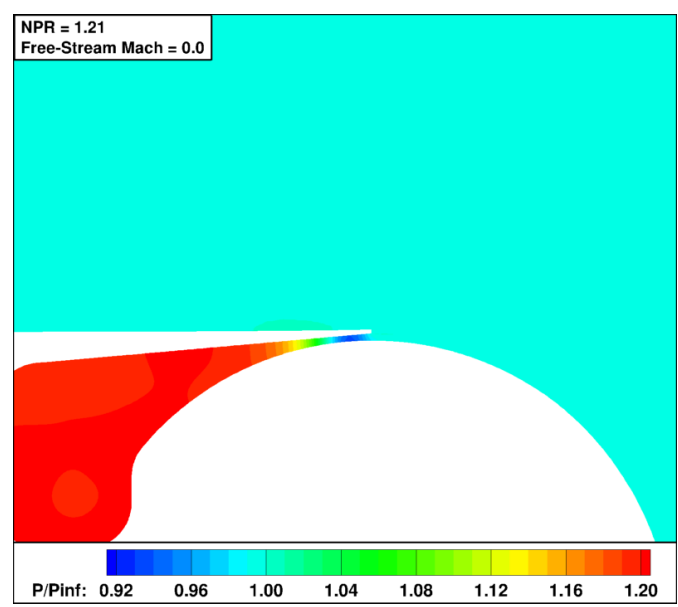

Figure 11. Contour of the static pressure ratio, $p / p_{\infty}$, from the CFD simulation for $\mathrm{NPR}=1.21$ with no external flow, $M_{\infty}=0$.

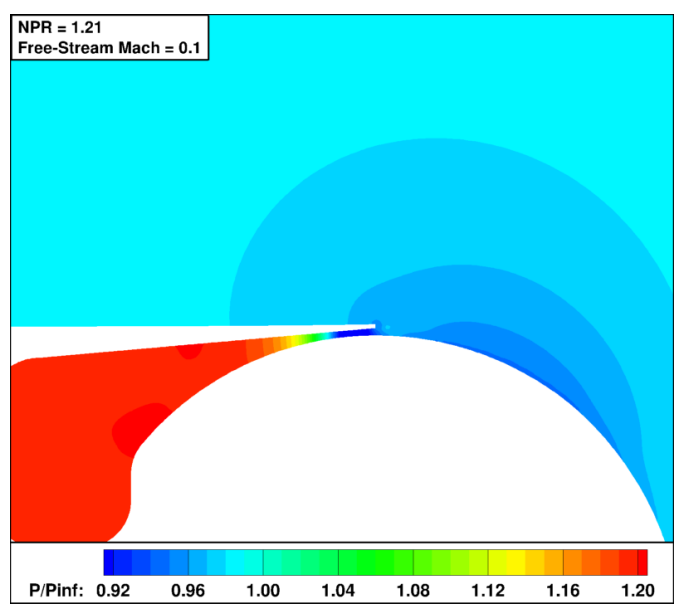

Figure 12. Contour of the static pressure ratio, $p / p_{\infty}$, from the CFD simulation for $\mathrm{NPR}=1.21$ with external flow, $M_{\infty}=0.1$.

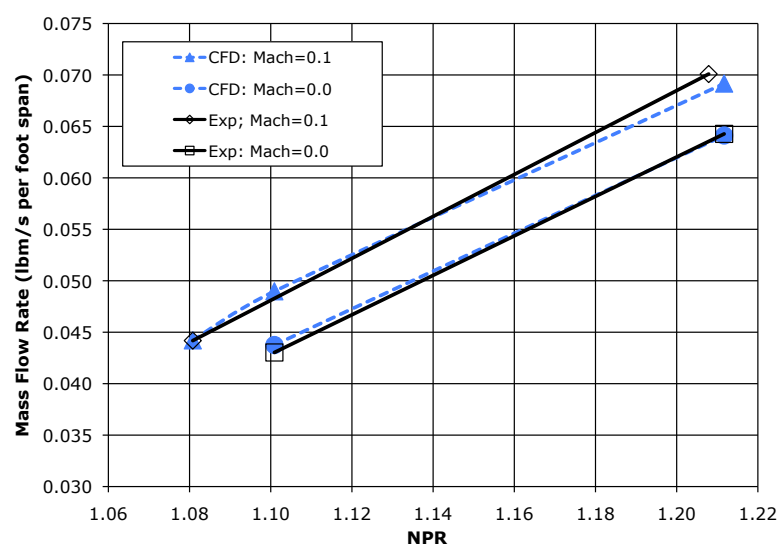

Figure 13. Mass flow rate as a function of NPR for the experiment and CFD with and without external flow.

correction and the experimental data has not been corrected for wall effects. Therefore, any downwash in the experiment has not been accounted for in the 2D CFD simulations, which will reduce this leading edge suction peak. Simulations modeling the tunnel walls will be discussed in section E below to address the effects of the downwash and tunnel walls on the lift coefficient and airfoil pressure distribution.

\section{2. $C_{\mu}=0.115$ Case}

Results for the higher blowing case of $C_{\mu}=0.115$ are shown in Fig. 15 for the $2 \mathrm{D}$ airfoil simulation. This figure compares the result for the SA and SST turbulence models with and without LMP and RCC. The SA model without RCC resulted in a $C_{L}$ value of 4.55 as compared to the experimental sectional $C_{L}$ value of 2.68 , which is a $70 \%$ over prediction of the lift coefficient. Turning on RCC with the SA model resulted in an improved $C_{L}$ of 3.90; however, this is still $46 \%$ larger than the experiment. Using both RCC and LMP with the SA turbulence model only reduced $C_{L}$ by less than $1 \%$ and resulted in a small addition of unsteadiness to the lift convergence history. Using the SST turbulence model resulted in an improved $C_{L}$ value of 3.84 and turning on RCC reduced the value of $C_{L}$ to 3.34. The SST model with RCC performed best; however, the predictions still show a $25 \%$ larger lift than the experiment. As in the $C_{\mu}=0.047$ blowing case, the $2 \mathrm{D}$ simulations for $C_{\mu}=0.115$ do not account for downwash on the model or tunnel wall effects by using an angle of attack correction. 


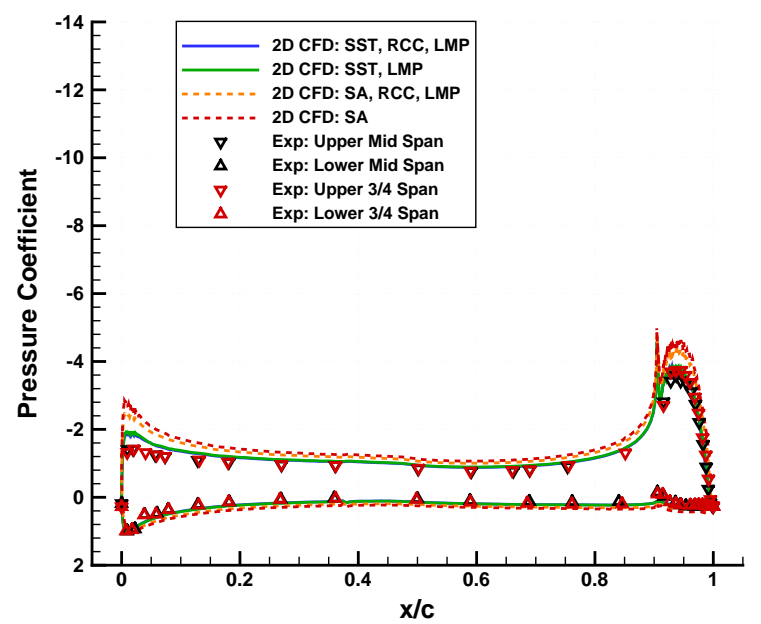

Figure 14. A comparison of the $C_{p}$ profiles using different turbulence models from the 2D CFD simulations to the experiment for $C_{\mu}=0.047$ with a freestream Mach number of 0.1. Experimental $C_{p}$ profiles are shown for the mid span of the model and at the $3 / 4$ span location.

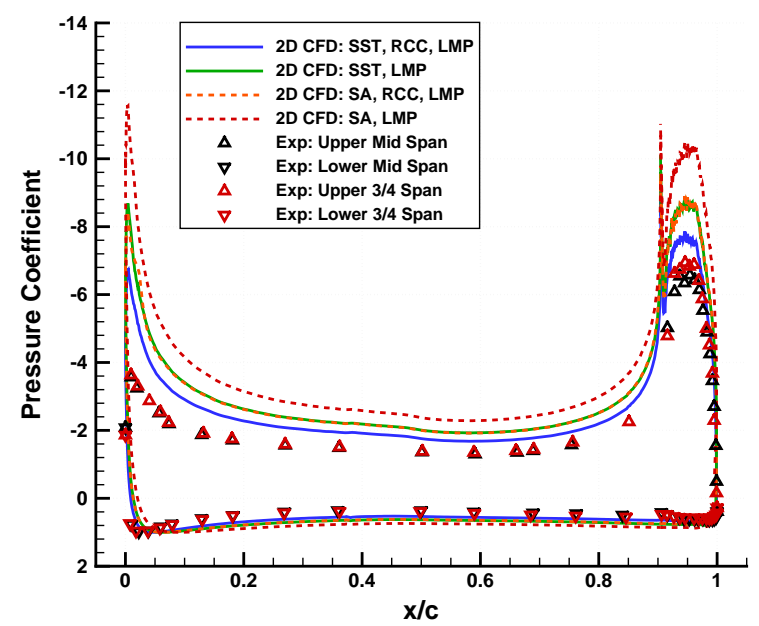

Figure 15. A comparison of the $C_{p}$ profiles using different turbulence models from the 2D CFD simulation to the experiment for $C_{\mu}=0.115$ with a freestream Mach number of 0.1. Experimental $C_{p}$ profiles are shown for the mid span of the model and at the $3 / 4$ span location.

\section{2D Grid Resolution Study}

A grid resolution study was made for the 2D 'free-air' case for the two $C_{\mu}$ blowing cases using coarse, medium, and fine grids. Table 2 summarizes the number of grid points for the three levels of grids where the medium grid is representative of the grid resolution of the 2D and 3D simulations. A fine grid was generated with a total of 2481 points around the airfoil with 801 points on the Coanda surface. The fine grid also had 801 points in the viscous direction, normal to the surface that extended a distance of $0.23 \mathrm{c}$. The grid spacing at the wall was set to $0.87 \times 10^{-7}$ for the fine grid so that the coarse grid would have $y^{+}<1$ at the wall. The fine grid case resulted in a total of approximately 1.8 million grid points for the body and background grids. The grids for the medium case were generated by removing every other grid point for the body grids resulting in a quarter of the grids points as the fine body grids. Similarly, the coarse grids were generated by removing every other grid point in the medium body grids. In all three cases the background grids were automatically generated by the flow solver where the resolution of the level 1 grid, which encloses the body grids, had a grid spacing of $0.0029 c$ for the fine grid case. This background spacing was doubled for the medium grid case to $0.0058 c$ and doubled again for the coarse grid case to $0.0116 c$.

The grid resolution cases were run using the SST turbulence model with RCC and without LMP. The results are given in Table 3 for $C_{\mu}$ values of 0.047 and 0.115 . The table also shows the Richardson Extrapolation (RE) results for the medium and coarse grids ( $\mathrm{RE} \mathrm{m}-\mathrm{c})$ and the fine and medium grids (RE $\mathrm{f}-\mathrm{m})$. The percentage difference from the $\mathrm{RE} \mathrm{f}-\mathrm{m}$ value is given in the $\% C_{L}$ column. The $0.115 C_{\mu}$ case shows good convergence where there is a $1.26 \%$ difference for the medium grid case, which is representative of the grid resolution used in the $2 \mathrm{D}$ and $3 \mathrm{D}$ simulations. The $0.047 C_{\mu}$ low blowing case also has good grid convergence with the medium grid resulting in a $0.85 \%$ difference.

\section{D. $C_{\mu}$ Calculations}

The CC airfoil performance is characterized as a function of the jet momentum coefficient, $C_{\mu}$, and is defined as:

$$
C_{\mu} \equiv \frac{\text { Thrust }}{q_{\infty} S}=\frac{\int\left(\rho_{j} \vec{V}_{j} \cdot \vec{n}\right)\left(\vec{V}_{j} \cdot \vec{n}\right) d A}{q_{\infty} S}
$$

where $\vec{V}_{j}$ is the jet velocity and $\vec{n}$ is the unit normal vector at the slot exit. For small scale CC experiments it is difficult to measure the jet velocity profile at the slot exit since the slot heights are very small. Therefore, 
Table 1. Summary of the 2D CFD lift coefficients for the SA and SST turbulence models with and without LMP and RC.

\begin{tabular}{|l|c|c|c|c|}
\cline { 4 - 5 } & \multirow{2}{*}{ LMP } & \multirow{2}{*}{ RCC } & \multicolumn{2}{|c|}{ Lift Coef., $C_{L}$} \\
\cline { 4 - 5 } & $C_{\mu}=0.047$ & $C_{\mu}=0.115$ \\
\hline \hline Exp. balance & - & - & 1.31 & 2.58 \\
Exp. 3/4 span; press. & - & - & 1.35 & 2.68 \\
\hline \multirow{5}{*}{ SA turb. model } & off & off & 2.03 & 4.55 \\
& on & off & 2.05 & 4.52 \\
& off & on & 1.86 & 3.90 \\
& on & on & 1.88 & 3.87 \\
\hline \multirow{2}{*}{ SST turb. model } & off & off & 1.58 & 3.84 \\
& on & off & 1.58 & 3.84 \\
& off & on & 1.56 & 3.36 \\
& on & on & 1.56 & 3.34 \\
\hline
\end{tabular}

Table 2. Summary of the mesh sizes for a 2D grid resolution study. Body grids consist of a main airfoil, circular trailing edge and internal nozzle grids. The total points for the body grids are in the body points column and the total number of points for the body and background grids are in the total points column.

\begin{tabular}{rcrrr}
\hline Grid & Main Airfoil & Circular TE & Body Points & Total Points \\
\hline \hline coarse & $421 \times 81$ & $201 \times 131$ & 94,317 & 168,319 \\
medium & $841 \times 161$ & $401 \times 261$ & 373,492 & 507,687 \\
fine & $1,681 \times 321$ & $801 \times 521$ & $1,486,527$ & $1,795,447$ \\
\hline
\end{tabular}

experimentally the integral in Eq. 1 is difficult to evaluate and in practice the $C_{\mu}$ values are approximated as:

$$
C_{\mu}=\frac{\dot{m}_{j} u_{j}}{q_{\infty} S}
$$

where the thrust is approximated as a product of the mass flow rate and a jet velocity $u_{j}$ normal to the slot exit. The value used for $u_{j}$ can be a source of much confusion and inconsistency between experiments and computations and can have a significant impact on the predicted $C_{\mu}$ values.

The $C_{\mu}$ value for the BART CC experiment was determined by measuring the mass flow rate, $\dot{m}$. The jet velocity at the slot exit, $u_{j}$, was then calculated using the following isentropic relations:

$$
u_{j}=\left(\frac{2}{\gamma-1} \gamma R T_{t, j}\left(1-\left(\frac{p_{j}}{p_{t, j}}\right)^{\frac{\gamma-1}{\gamma}}\right)\right)^{1 / 2}
$$

where $p_{t, j}$ is the total pressure measured inside the second plenum and $p_{j}$ is the static pressure at the jet exit. This static pressure is typically approximated as the freestream static pressure, $p_{\infty}$. However, the actual static pressure at the slot exit for CC blowing airfoils is much lower than freestream and results in an under prediction of the jet exit velocity.

Therefore, using the isentropic relations the jet velocity can be calculated by measuring the nozzle pressure ratio, $N P R=p_{\infty} / p_{t, j}$ and the total jet temperature. The isentropic relations assume that there are no heat transfer and frictional effects. While heat transfer effects are assumed to be small in the experiment, the frictional effects (i.e., boundary layers) for a slot of this size are not insignificant.

A comparison of the $C_{\mu}$ values between the experiment and CFD are given in Table 4 for the two blowing cases. These comparisons show that the $C_{\mu}$ values based on the isentropic $u_{j}$, assuming $p_{j} \approx p_{\infty}$, are comparable between the experiment and the CFD using the same approach. The table also shows that the mass flow rate per unit span are nearly identical between the CFD and experiment. 
Table 3. Summary of the lift coefficient results for the grid resolution study. The percentage differences are from changes from the Richardson Extrapolation of the fine and medium (RE f-m) grids.

\begin{tabular}{r|cc|cc}
\hline & \multicolumn{2}{|c|}{$C_{\mu}=0.047$} & \multicolumn{2}{c}{$C_{\mu}=0.115$} \\
Grid & $C_{L}$ & $\% C_{L}$ & $C_{L}$ & $\% C_{L}$ \\
\hline \hline coarse & 1.5574 & 0.99 & 3.3198 & 2.42 \\
medium & 1.5596 & 0.85 & 3.3594 & 1.26 \\
fine & 1.5697 & 0.21 & 3.3914 & 0.31 \\
RE m-c & 1.5604 & 0.80 & 3.3726 & 0.87 \\
RE f-m & 1.5730 & - & 3.4021 & - \\
\hline Exp. (press.) & 1.35 & & 2.68 \\
\hline
\end{tabular}

Table 4. A comparison of $C_{\mu}$ values and jet exit velocities, $u_{j}$, between the experiment and CFD 2D simulations for two jet blowing cases.

\begin{tabular}{|l|c|c||c|c|}
\hline \multirow{2}{*}{} & \multicolumn{2}{|c||}{$C_{\mu}=0.047$ Case } & \multicolumn{2}{c|}{$C_{\mu}=0.115$ Case } \\
\cline { 2 - 5 } & Exp. & CFD & Exp. & CFD \\
\hline $\mathrm{NPR}$ & 1.081 & 1.081 & 1.208 & 1.208 \\
$T_{t, \infty} / T_{t, j}$ & 0.990 & 0.990 & 0.987 & 0.987 \\
$\dot{m}(\mathrm{lbm} / \mathrm{s} / \mathrm{ft})$ & 0.0437 & 0.0435 & 0.0693 & 0.0680 \\
\hline$C_{\mu}\left(u_{j}\right.$ isentropic, $\left.p_{j} \approx p_{\infty}\right)$ & 0.047 & 0.047 & 0.115 & 0.114 \\
$C_{\mu}$ (integrated) & - & 0.050 & - & 0.120 \\
$C_{\mu}\left(u_{j}\right.$ mean $)$ & - & 0.045 & - & 0.109 \\
$C_{\mu}\left(u_{j}\right.$ peak $)$ & - & 0.055 & - & 0.130 \\
\hline$u_{j}$ isentropic, $p_{j} \approx p_{\infty}(f t / s)$ & 372 & 374 & 576 & 577 \\
$u_{j}$ mean $(f t / s)$ & - & 353 & - & 547 \\
$u_{j}$ peak $(f t / s)$ & - & 436 & - & 659 \\
$u_{j}$ peak, isentropic, $p_{j} \approx p_{l i p}(f t / s)$ & - & 431 & - & 648 \\
\hline
\end{tabular}

The actual value of $C_{\mu}$ can be computed using the CFD solution by integrating Eq. 1 across the jet exit. For the low blowing case the actual $C_{\mu}$ value from the CFD solution is 0.050 as compared to the approximated value of 0.047 using the isentropic jet velocity. For the higher blowing case the integrated $C_{\mu}$ from the CFD is 0.120 as compared to the isentropic value of $C_{\mu}=0.114$. In both blowing cases, the $C_{\mu}$ values based on measuring the mass flow rate and the isentropic $u_{j}$ resulted in a $5-6 \%$ lower value of the thrust coefficient as compared to the actual $C_{\mu}$. Table 4 also shows that the variation in $C_{\mu}$ values base on the mean tend to under predict $C_{\mu}$ while using the peak velocity the $C_{\mu}$ values are over predicted. Based on these comparisons, it is important when comparing $C_{\mu}$ values between experiment and CFD results that the values of $C_{\mu}$ are computed using a similar approach. Therefore, if the experiment is calculating $C_{\mu}$ based on a measured mass flow rate and $u_{j}$ from isentropic relations, then the CFD should compute $C_{\mu}$ using the same approach.

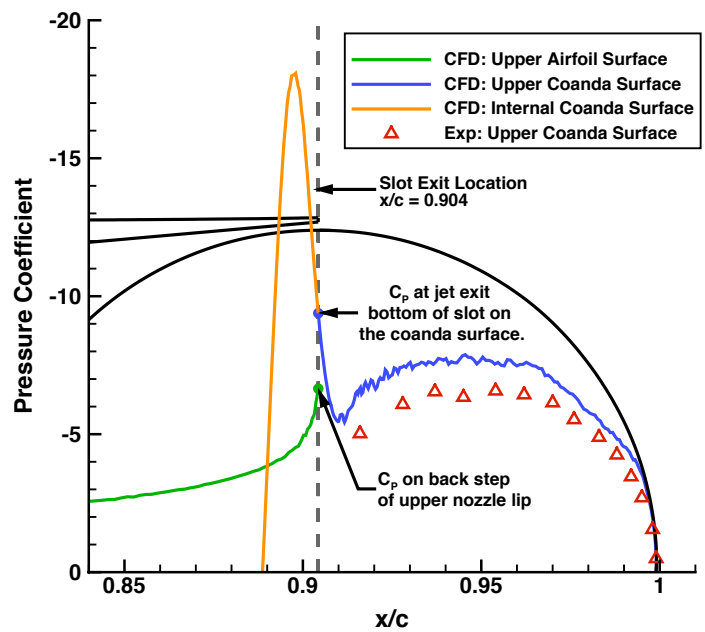

Figure 16. Surface $C_{p}$ near the slot exit for the $C_{\mu}=0.115$ blowing case. 


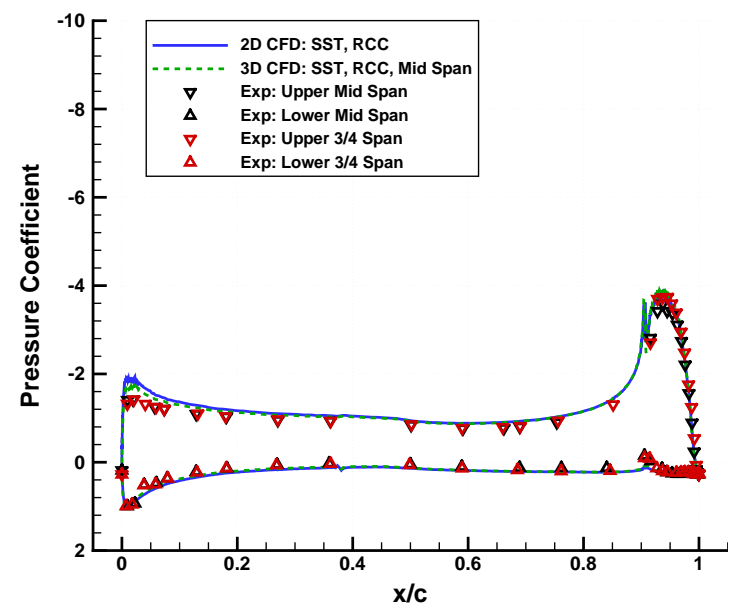

Figure 17. A comparison of the $2 \mathrm{D}$ and $3 \mathrm{D}$ CFD results to the experimental $C_{p}$ profile data for $C_{\mu}=0.047$ with a freestream Mach number of 0.1. Experimental $C_{p}$ profiles are shown for the mid span of the model and at the $3 / 4$ span location. The $C_{p}$ profile for the $3 \mathrm{D}$ CFD results are from the mid span of the model.

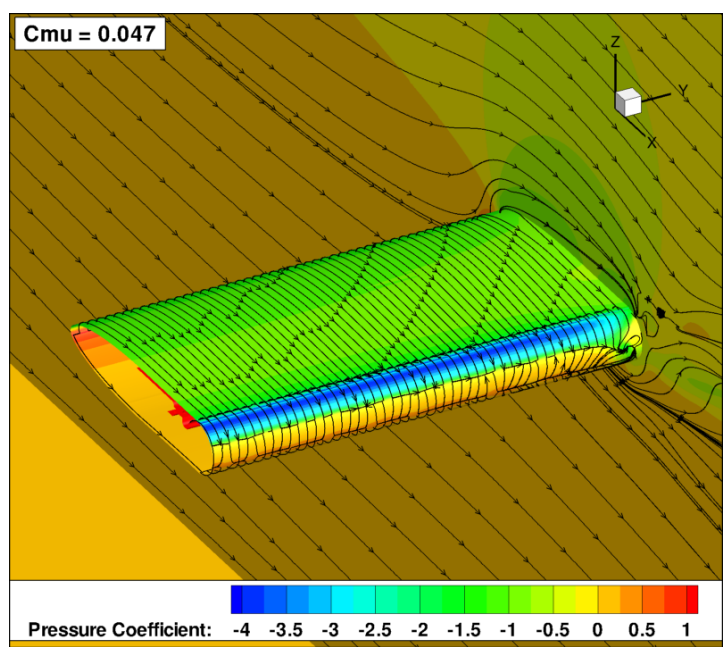

Figure 18. Contour plots of $C_{p}$ on the model and tunnel walls with surface restricted streamlines for the 3D CFD simulation at $C_{\mu}=0.047$. Shown is the CC model from the tunnel side wall to the mid span of the model where the plane of symmetry boundary condition was imposed.

Table 4 also shows a calculation of the jet peak velocity using the isentropic relations and the static pressure on the back face of the nozzle upper lip, $p_{l i p}$, from the CFD solution. Figure 16 shows a close-up view of the $\mathrm{CC}$ trailing edge and blowing slot with a plot of $C_{p}$ on the Coanda surface for the CFD and experiment. This figure also shows the $C_{p}$ values inside the plenum lower surface and on the external surface of the airfoil, upstream of the slot. From this figure it can be seen that while the experimental pressure measurements downstream of the nozzle are close to the slot, they are not appropriate for computing the peak jet velocity since the pressure increases rapidly downstream of the slot. For the $C_{\mu}=0.047$ CFD simulation, the jet peak velocity using $p_{l i p}$ and the isentropic relations is $431 \mathrm{ft} / \mathrm{s}$ as compared to the peak value of $436 \mathrm{ft} / \mathrm{s}$ from the CFD simulation. In this calculation the total pressure in the plenum was used assuming no losses in the jet core, where the peak jet velocity is being calculated. For the $C_{\mu}=0.115$ CFD simulation, the jet peak value using $p_{\text {lip }}$ and the isentropic relations is $648 \mathrm{ft} / \mathrm{s}$ as compared to the peak value of $659 \mathrm{ft} / \mathrm{s}$ from the CFD simulation. Based on these calculations of $u_{j}$, using the local static pressure on the nozzle lip and the isentropic relations, the peak velocity of the jet can be predicted within approximately $2 \%$ as compared to approximately $13 \%$ using the freestream static pressure. Therefore, it is recommended for $\mathrm{CC}$ experiments to measure the local static pressure when estimating the peak jet velocity when comparing to CFD. That being said, it is recognized that this type of static pressure measurement near the slot exit can be very difficult to acquire accurately.

\section{E. 3D CFD Simulations}

The difficulty in comparing 2D "free-air" CFD simulations to the "2D" wind tunnel experiments is that the flow field for the experiment is inherently three dimensional with wall effects. The wall effects are dependent on many factors, one of them is the model size as compared to the size of the tunnel and another is the amount of lift generated by the airfoil. For circulation control problems, the goal is to generate very large lift coefficients, which consequently increase the wall effects. Another issue is that it is difficult to build models with very small blowing slots and to be able to set and measure the slots sizes accurately. Also measuring the flow accurately near the slot becomes challenging as the slots decrease in size. This results in a trade off between keeping the model to some reasonable size, minimizing wall effects, and making sure the model is large enough for accurate measurements at the slot.

One of the ways to account for the tunnel wall effects is to experimentally estimate an angle of attack correction and tunnel $q$ correction. ${ }^{16}$ These wall corrections have been shown to work well but become difficult to derive for high lift models. Also the validity of angle of attack and $q$ corrections needs to be questioned for models with very high lift coefficients and low aspect ratios as is the case for this test data. It 


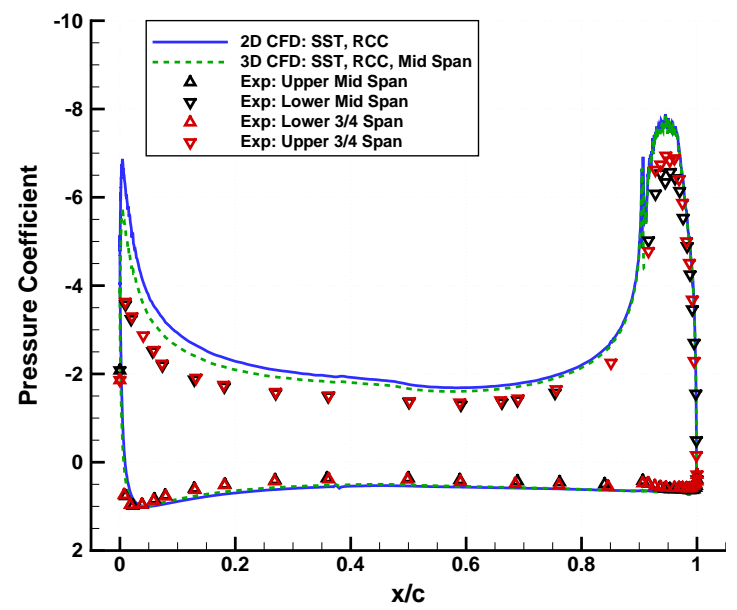

Figure 19. A comparison of the $2 D$ and $3 D$ CFD results to the experimental $C_{p}$ profile data for $C_{\mu}=0.115$ with a freestream Mach number of 0.1. Experimental $C_{p}$ profiles are shown for the mid span of the model and at the $3 / 4$ span location. The $C_{p}$ profile for the $3 \mathrm{D}$ CFD results are from the mid span of the model.

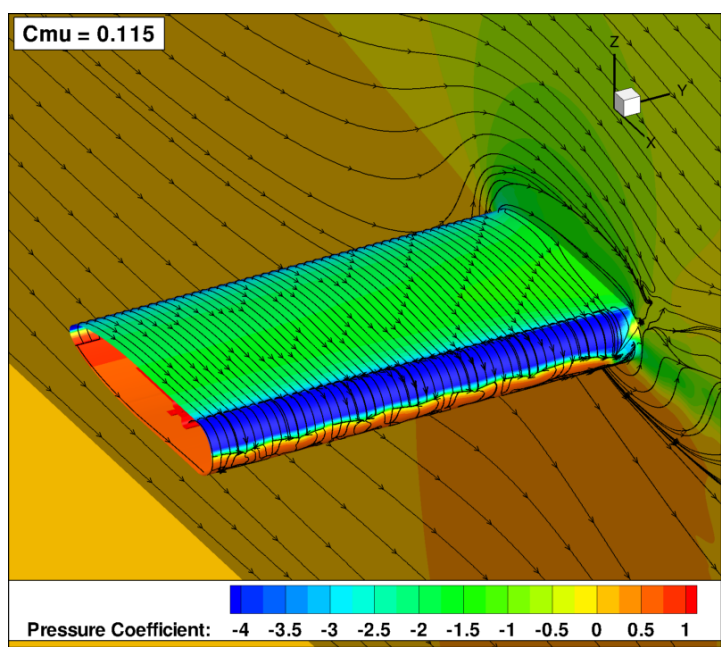

Figure 20. Contour plots of $C_{p}$ on the model and tunnel walls with surface restricted streamlines for the 3D CFD simulation at $C_{\mu}=0.115$. Shown is the CC model from the tunnel side wall to the mid span of the model where the plane of symmetry boundary condition was imposed.

also becomes difficult to isolate wall effects from modeling issues when CFD and experiments do not match.

Another way to address the wall effects issue, and the approach taken in this investigation, is to perform a 3D CFD simulation of the model and the tunnel walls and actually simulate the wind tunnel experiment. While this sounds straight forward, additional challenges like increased cost due to the large number of grid points required and the difficulty of modeling the wall juncture vortex accurately arise.

\section{1. $C_{\mu}=0.047$ Case}

From the 2D simulations, it was found that the SST turbulence model with RCC performed best at matching the surface pressures at the jet exit and was therefore used in the 3D simulations. Simulating the model and tunnel for $C_{\mu}=0.047$ resulted in a $C_{L}$ of 1.47 for the entire model with a sectional $C_{L}$ at midspan of 1.53. A summary of the lift results are given in Table 5 showing that the sectional $C_{L}$ at the midspan for the 3D CFD simulation results in a $1 \%$ lower value as compared to the $2 \mathrm{D}$ CFD. Therefore, the wall effects on the midspan $C_{L}$ are shown to be small for the CFD simulations as might be expected for this low blowing case.

A comparison of the $C_{p}$ profiles is given in Fig. 17 showing a slight difference in the leading edge suction pressure peak between the $2 \mathrm{D}$ and 3D midspan CFD predictions with no difference at the jet exit and Coanda surface. This difference in the leading edge peak $C_{p}$ values is attributed to the downwash effect in the 3D simulations, reducing the effective angle of attack on the model thus producing a smaller leading edge suction pressure peak. While the $3 \mathrm{D}$ results are improved the effects of the walls does not improve the over prediction of the lift as seen in the 2D CFD cases. Surface $C_{p}$ contours and streamlines are shown in Fig. 18 highlighting the effects of the juncture flow on the tunnel walls

Table 5. Summary of Lift coefficient, $C_{L}$, between experiment and 2D and 3D CFD simulations. Experiment data shown is from the balance for the entire model and a sectional $C_{L}$ integrated from pressures near the $3 / 4$ span of the model at $y / b=0.732$.

\begin{tabular}{|l|c|c|}
\cline { 2 - 3 } \multicolumn{1}{c|}{} & \multicolumn{2}{c|}{ Lift Coef., $C_{L}$} \\
\cline { 2 - 3 } & $C_{\mu}=0.047$ & $C_{\mu}=0.115$ \\
\hline \hline Exp. balance & 1.31 & 2.58 \\
Exp. 3/4 span; press. & 1.35 & 2.68 \\
\hline 2D CFD & 1.56 & 3.36 \\
3D CFD; model & 1.47 & 3.09 \\
3D CFD; midspan & 1.53 & 3.21 \\
\hline
\end{tabular}
and model. 


\section{2. $C_{\mu}=0.115$ Case}

The $C_{\mu}=0.115$ case has a much higher $C_{L}$ and is expected to have larger wall effects as compared to the lower blowing case. The 3D CFD simulation for the $C_{\mu}=0.115$ case resulted in a $C_{L}=3.09$ for the whole model with a sectional $C_{L}$ value of 3.21 at the midspan of the model. This case only shows a $4 \%$ decrease in the midspan $C_{L}$ as compared to the 2D CFD $C_{L}$ of 3.36. Therefore, the $C_{\mu}$ of 0.115 also shows a small effect of the walls on the midspan sectional lift coefficient as was seen in the low blowing case.

A comparison of the $C_{p}$ profiles between the CFD and the experiment is made in Fig. 19. This comparison shows a difference in the leading edge suction pressure peak between the 2D and 3D CFD results resulting in a lower lift prediction for the latter. This comparison also shows no difference in the $C_{p}$ values at the jet exit and Coanda surface for the 3D simulation. However, the 3D CFD is still over predicting the lift sectional coefficient at the midspan by $20 \%$ showing that the CFD is not completely capturing the correct physics on the Coanda surface as in the $2 \mathrm{D}$ simulations.

Figure 20 shows the surface contours and streamlines for the 3D CFD simulation and the effects of the extent of the juncture flow on the model and tunnel walls. This figure also reveals some interesting waviness of the separation location on the Coanda surface.

\section{Tunnel Wall Pressures}

The tunnel wall pressures were measured on the centerline of the walls opposite of the upper and lower surface of the CC model. The wall pressure taps begin near the tunnel contraction and extend down the test section and end just before the tunnel diffuser. Figure 21 compares the upper and lower wall pressures for the baseline, no blowing condition for the CFD and experiment. The plot shows very low $C_{p}$ values upstream and downstream of the model as expected since there is no lift on the model at this condition. There is a large jump in the tunnel wall $C_{p}$ values about 4 chord lengths downstream from the trailing edge, which corresponds to a $2 \mathrm{in}$. wide slot on the ceiling at $x / c=4$ that extends across the ceiling and ends $6.5 \mathrm{in}$. from each of the side walls. This slot was used for a 7 -hole probe rake to measure the downstream wake flow; however, it has resulted in contamination of the wall pressures.

The wall pressures for the two blowing conditions investigated in this paper are shown in Figs. 22 and 23. These two figures show how the 3D CFD simulations are matching the entrance $C_{p}$ values of the experiment. As a result of the over prediction in the lift, the wall pressure at the same blowing conditions are also over predicted. Downstream of the model, near the ceiling slot, it is difficult to compare the wall pressure since the CFD is not modeling the open rake slot in the wind tunnel. Figures 24 and 25 show that by subtracting the baseline wall pressures data and plotting, $\Delta C_{p}=C_{p}-C_{p, \text { Baseline }}$, that the pressure jump associated with the open rake slot in the tunnel is removed, improving the comparison with the CFD. Overall the general shape of the wall $C_{p}$ profiles are similar between the experiment and the CFD. The peak values do not match between the CFD and experiment since the CFD over predicts the model lift.

\section{Spanwise Model Pressures}

A spanwise row of pressure taps was placed on the upper surface of the model at $x / c=0.69$ in order to measure the uniformity of the flow along the span. Figure 26 shows the spanwise pressure profile for the experiment and the CFD at two blowing conditions. Since the CFD simulation was performed with a plane of symmetry along the centerline of the CC model, the $C_{p}$ distribution has been reflected about the mid span of the model making it implicitly symmetric. The base of the $\mathrm{CC}$ model is at a span location of $z=0 \mathrm{in}$. where the model extends through the wind tunnel floor and is mounted on a force and moment balance. The model is mounted on a circular base plate that has an overlapping region on the edge of the plate, reducing 


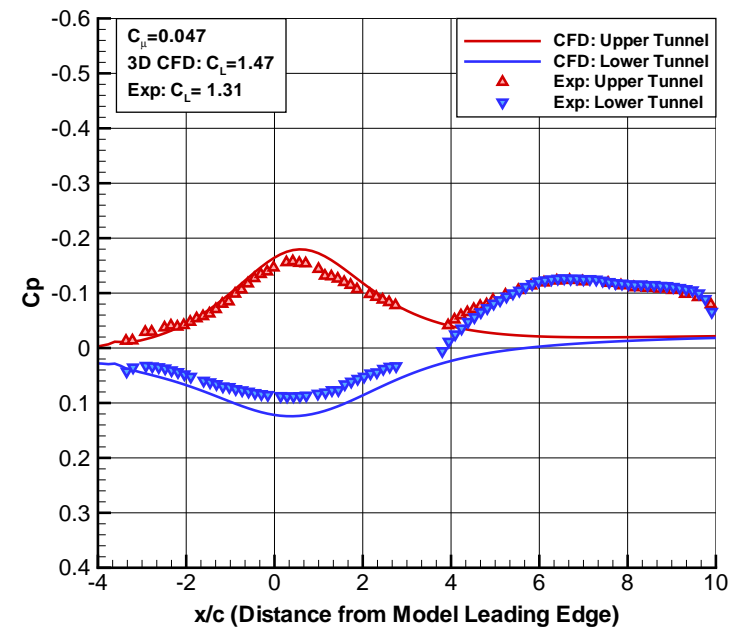

Figure 22. Tunnel wall centerline pressures for the walls on the upper and lower side of the airfoil for the $C_{\mu}=0.047$ blowing case.

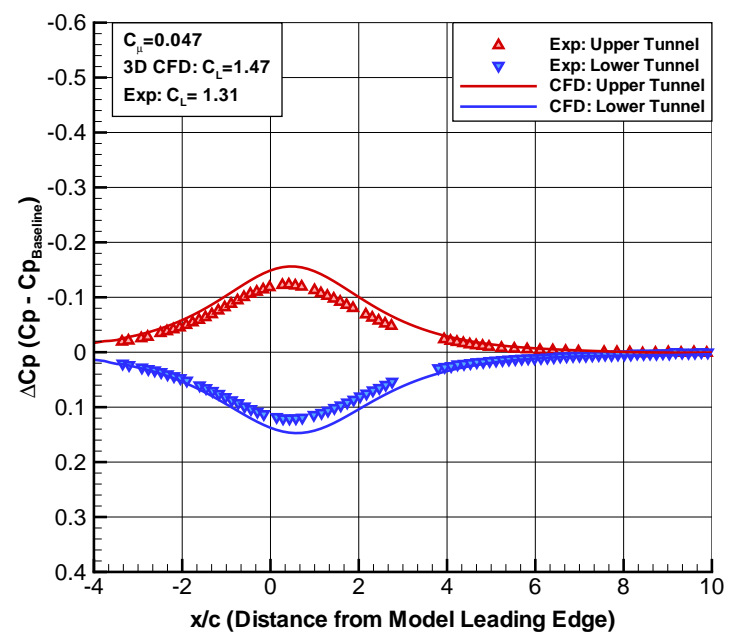

Figure 24. Tunnel wall centerline $\Delta C_{p}$ values on the upper and lower side of the airfoil for the $C_{\mu}=0.047$ blowing case.

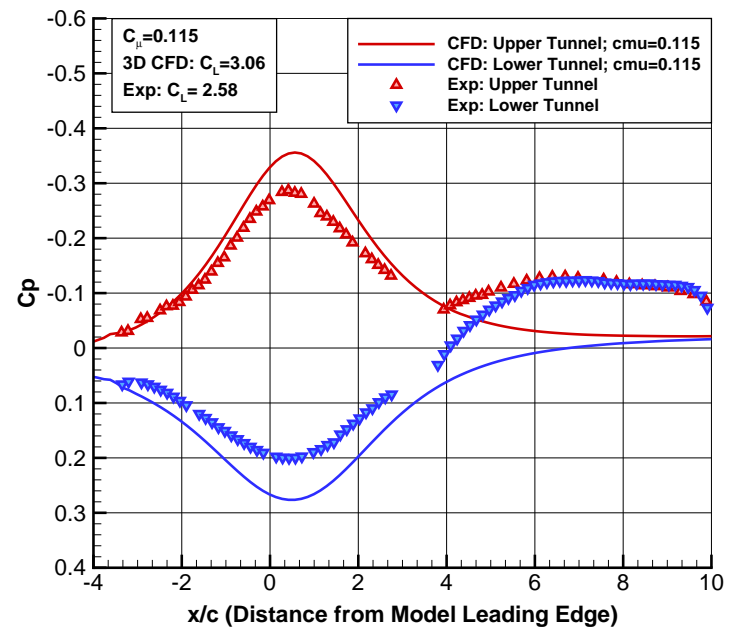

Figure 23. Tunnel wall centerline pressures for the walls on the upper and lower side of the airfoil for the $C_{\mu}=0.115$ blowing case.

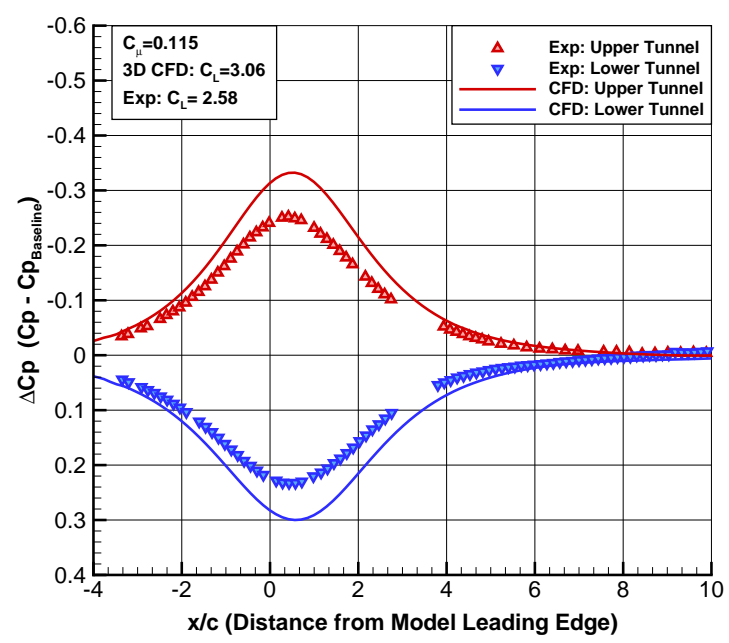

Figure 25. Tunnel wall centerline $\Delta C_{p}$ values on the upper and lower side of the airfoil for the $C_{\mu}=0.115$ blowing case.

the external flow into the test section. The tip of the CC model is at a span location of $z=28 \mathrm{in}$. and has a small gap between the model and the wall. The spanwise profiles from the experiment are non-symmetric with an increase in pressure near the base of the model versus the tip. The figure shows that for $z<15 \mathrm{in}$. the $C_{p}$ values are higher compared with data at $z>15 \mathrm{in}$. This increase in $C_{p}$ near the base indicates that the model may have a reduction of lift as compared to the other half of the model. The ratio of the sectional $C_{L}$ near the $3 / 4$ span location to the model balance $C_{L}$ is 1.04 for the experiment at $C_{\mu}$ of 0.115 . The ratio of the midspan sectional $C_{L}$ to model $C_{L}$ for the CFD is also 1.04, indicating that the loss of lift seen in the spanwise pressure for the experiment is very small and is not having a significant impact on the model lift. At this time it is unknown as to the source of the non-symmetric $\mathrm{Cp}$ distribution and requires further investigation. The profiles of $C_{p}$ from near the centerline at $z=15 \mathrm{in}$. to the tip at $z=28 \mathrm{in}$. are similar in character between the experiment and the CFD results. The CFD simulations, at matching $C_{\mu}$ values, also show that the CFD significantly over predicts the $C_{p}$ values, which is consistent with the CFD over predicting the total model lift.

The spanwise lift distribution is given in Fig. 27 for the 3D CFD simulations at the two blowing conditions. This figure shows that the lift distribution is fairly flat for the two blowing cases over most of the model; however, there is a significant loss of lift near the tunnel walls. This loss of lift near the wall results in a difference of the lift coefficient at the midspan as compared to the entire model and is more pronounced for the higher blowing case, as expected. 


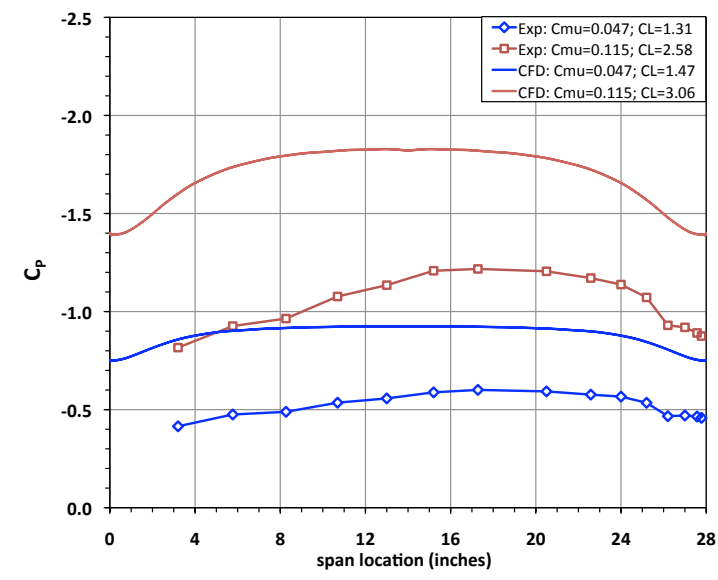

Figure 26. Comparison of the spanwise $C_{p}$ distribution between the experiment and CFD for two blowing conditions. The spanwise pressure ports are located at the $x / c=0.69$ station on the upper surface of the airfoil.

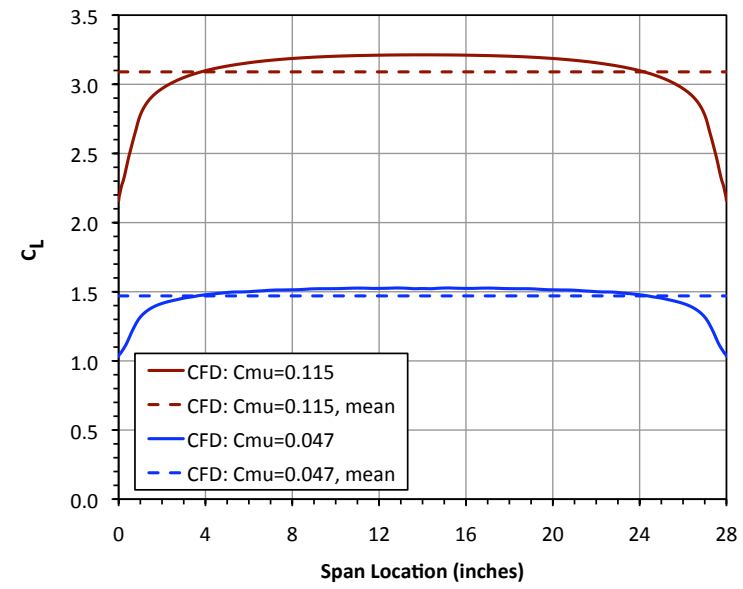

Figure 27. CFD spanwise $C_{L}$ distribution for $C_{\mu}=$ 0.115 .

\section{Summary}

RANS simulations of a circulation control model used in recent wind tunnel experiments conducted at the NASA Langley BART have been performed. The initial CFD investigation started by comparing the slot velocity profiles to experimental bench top hot-wire measurements. These comparisons showed good agreement to the 2D CFD simulation with a quiescent flow condition at matching NPR values. Using the same NPR for a freestream Mach number of 0.1 resulted in a higher peak jet velocity due to the reduced static pressure near the exit of the nozzle when lift is generated by the oncoming freestream flow. These results showed that the peak jet exit velocity, that would exist in a wind tunnel test or in flight, cannot be inferred by direct measurement on the bench top with no external flow and should to be measured in the wind tunnel. Based on CFD simulations, the jet profile shape did not change with external flow, suggesting that the jet exit profiles can be measured on the bench top and only the peak jet velocity needs to be measured in the tunnel, thus reducing the complexity of the wind tunnel test.

Evaluation of the CFD results showed that the freestream static pressure is not appropriate in calculating the jet exit velocity using isentropic relations. It was discovered that a static pressure measurement near the slot exit on the upper lip of the nozzle can be used to calculate the peak jet exit velocity within $2 \%$. However this measurement can be difficult due to the size of the slot and the rapid change in the static pressure downstream of the slot. A static pressure measurement on the back step of the upper nozzle lip is experimentally possible and was shown to be a good location for the calculation of the jet peak velocity based on the CFD simulations.

Simulations in 2D were performed to evaluate the SST and SA turbulence models and the effect of RCC and LMP on the prediction of the airfoil sectional lift coefficient. The $2 \mathrm{D}$ simulations showed that the SA turbulence model greatly over predicted the lift as compared to the SST model. In fact, the 2D CFD simulation using the SA turbulence model for $C_{\mu}=0.115$ over predicted $C_{L}$ by $70 \%$. Including the RCC to the turbulence models improved the CFD predictions where the SST model with the RCC gave the best results. However the SST with RCC still over predicted sectional $C_{L}$ by $25 \%$ without using wall corrections. Using LMP did not show a significant effect on $C_{L}$ but introduced a small amount of unsteadiness to the lift convergence history.

The effects of the tunnel walls were addressed by performing 3D CFD simulations modeling the wind tunnel experiment. These simulations showed only a small effect of the tunnel walls on the sectional lift at the midspan of the model. The CFD results showed a $4 \%$ difference in the midspan sectional $C_{L}$ values for the $C_{\mu}=0.115$ case. Therefore, the wall effects for the two blowing cases investigated in this paper have been shown to be very small compared to the over prediction of the lift by the RANS flow solver. 


\section{References}

1 "Proceedings of the 2004 NASA/ONR Circulation Control Workshop," Proceedings of the 2004 NASA/ONR Circulation Control Workshop, edited by G. S. Jones and R. D. Joslin, NASA/CP 2005-213509, Hampton, Virginia, 2004.

${ }^{2}$ Rumsey, C. L. and Nishino, T., "Numerical Study Comparing RANS and LES Approaches on a Circulation Control Airfoil," 49th AIAA Aerospace Sciences Meeting, American Institute of Aeronautics and Astronautics, Reston, VA (submitted for publication).

${ }^{3}$ Jones, G. S., Lin, J. C., Allan, B. G., Milholen, W. E., Rumsey, C. L., and Swanson, R. C., "Overview of CFD Validation Experiments for Circulation Control Applications at NASA," RAES-566-8A1, International Powered Lift Conference, London, July 2008.

${ }^{4}$ Englar, R., Jones, G. S., Allan, B. G., and Lin, J. C., "2-D Circulation Control Airfoil Benchmark Experiments Intended for CFD Code Validation," AIAA Paper 2009-902, January 2009.

${ }^{5}$ Englar, R., Gaeta, R., Jones, G. S., and Milholen, W., "Recent Experimental Development of Circulation Control Airfoils and Pneumatic Powered-Lift Systems," AIAA Paper 2010-0345, January 2010.

${ }^{6}$ Buning, P. G., Jespersen, D. C., Pulliam, T. H., Klopfer, W. M., Chan, W. M., Slotnick, J. P., Krist, S. E., and Renze, K. J., "OVERFLOW User's Manual Version 1.8m," Tech. rep., NASA Langley Research Center, 1999.

${ }^{7}$ Jespersen, D., Pulliam, T., and Buning, P., "Recent Enhancements to OVERFLOW," AIAA paper 97-0644, January 1997.

${ }^{8}$ Pulliam, T. H. and Chaussee, D. S., "A Diagonal Form of an Implicit Approximate-Factorization Algorithm," Journal of Computational Physics, Vol. 39, February 1981, pp. 347-363.

${ }^{9}$ Steger, J. L., Dougherty, F. C., and Benek, J. A., "A Chimera Grid Scheme," Advances in Grid Generation, edited by K. N. Ghia and U. Ghia, Vol. 5 of FED, ASME, New York, NY, 1983.

${ }^{10}$ Murphy, K., Buning, P., Pamadi, B., Scallion, W., and Jones, K., "Status of Stage Separation Tool Development for Next Generation Launch Technologies," AIAA Paper 2004-2595, June 2004.

${ }^{11}$ Menter, R. F., "Two-Equation Eddy-Viscosity Turbulence Models for Engineering Applications," AIAA Journal, Vol. 32, No. 8, 1994, pp. 1598-1605.

${ }^{12}$ Spalart, P. and Allmaras, S. R., "One-Equation Turbulence Model for Aerodynamic Flows," AIAA Paper 1992-0439, 1992.

${ }^{13}$ Shur, M., Strelets, M., Travin, A., and Spalart, P., "Turbulence Modeling in Rotating and Curved Channels: Assessing the Spalart-Shur Correction," AIAA Journal, Vol. 38, No. 5, May 2000, pp. 784-792.

${ }^{14}$ Chan, W. M. and Gomez, R. J., "Advances in Automatic Overset Grid Generation Around Surface Discontinuities," AIAA Paper 99-3303, July 1999.

${ }^{15}$ Sellers, W. L. and Kjelgaard, S. O., "The Basic Aerodynamics Research Tunnel - A Facility Dedicated to Code Validation," AIAA Paper 1988-1997, May 1988.

${ }^{16}$ Rae, W. H. and Pope, A., Low-Speed Wind Tunnel Testing, John Wily \& Sons, New York, 1984. 